\title{
Usefulness of Cathepsin S to Predict Risk for Obstructive Sleep Apnea among Patients with Type 2 Diabetes
}

\author{
Wanwan Wen, ${ }^{1}$ Haili Sun, ${ }^{2}$ Yunxiao Yang, ${ }^{1}$ Yifan Jia, ${ }^{1}$ Fang Fang, ${ }^{2}$ Yanwen Qin, ${ }^{3}$ \\ Ming Zhang $\mathbb{D}^{1}{ }^{1}$ and Yongxiang Wei $\mathbb{D}^{2}$ \\ ${ }^{1}$ Department of Cardiology, Beijing Anzhen Hospital, Capital Medical University, Beijing 100029, China \\ ${ }^{2}$ Department of Otolaryngology Head \& Neck Surgery, Key Laboratory of Upper Airway Dysfunction-Related \\ Cardiovascular Diseases, Beijing Institute of Heart, Lung and Blood Vessel Diseases, Beijing Anzhen Hospital, \\ Capital Medical University, Beijing 100029, China \\ ${ }^{3}$ Beijing Institute of Heart, Lung and Blood Vessel Diseases, Beijing Anzhen Hospital, Capital Medical University, \\ Beijing 100029, China
}

Correspondence should be addressed to Ming Zhang; zhangming2279@hotmail.com and Yongxiang Wei; weiyongxiang@vip.sina.com

Received 19 March 2020; Revised 20 May 2020; Accepted 18 August 2020; Published 26 September 2020

Academic Editor: Kishore Chaudhry

Copyright () 2020 Wanwan Wen et al. This is an open access article distributed under the Creative Commons Attribution License, which permits unrestricted use, distribution, and reproduction in any medium, provided the original work is properly cited.

\begin{abstract}
Background. Obstructive sleep apnea (OSA) was highly prevalent in patients with type 2 diabetes (T2D). Cathepsin S (CTSS), a cysteine protease, is involved in the inflammatory activity in T2D and hypoxia conditions. The aim of the study was to evaluate whether CTSS could be involved in the inflammatory reaction of OSA in patients with T2D. Methods. We included 158 participants in this study matched for age, gender, and body mass index in 4 groups (control, non-OSA\&T2D, OSA\&non-T2D, and OSA\&T2D). After overnight polysomnography, we collected the clinical data including anthropometrical characteristics, blood pressure, and fasting blood samples in the morning. Plasma CTSS concentration was evaluated using the human Magnetic Luminex Assay. Results. Compared with the control group, both the non-OSA\&T2D group and the OSA\&non-T2D group showed higher CTSS levels. Plasma CTSS expression was significantly increased in subjects with OSA\&T2D compared to subjects with nonOSA\&T2D. The OSA\&T2D group had higher CTSS levels than the OSA\&non-T2D group, but there were no statistically significant differences. Plasma CTSS levels showed significant correlation with the apnea-hypopnea index (AHI) $(r=0.559, P<0.001)$ and plasma fasting blood glucose $(r=0.427, P<0.001)$. After adjusting confounding factors, plasma CTSS levels were independently associated with the AHI (Beta: 0.386, 95\% confidence intervals (CI): 21.988 to $57.781 ; P<0.001$ ). Furthermore, we confirmed the higher pinpoint accuracy of plasma CTSS in the diagnosis of OSA (area under the curve: 0.868). Conclusions. Plasma CTSS expression was significantly elevated in the OSA\&T2D group and was independently associated with the AHI; it could be a biomarker with a positive diagnostic value on diagnosing OSA among patients with T2D.
\end{abstract}

\section{Introduction}

Obstructive sleep apnea (OSA) is a common disease that is characterized by repetitive collapse of the upper airway during sleep resulting in snoring, sleep disruption, nocturnal chronic intermittent hypoxia $(\mathrm{CIH})$, and oxidative stress, which cause endothelial dysfunction and inflammatory reaction [1-3]. Previous studies have reported that OSA severity is being gradually recognized as a possible cause of glucose intolerance, insulin resistance, and diabetes $[4,5]$. Moreover, it has also been reported that diabetes could increase the predisposition for OSA [6, 7]. Furthermore, multiple epidemiological studies showed that around 50\%-90\% of patients with type 2 diabetes (T2D) have OSA; thus, screening for OSA could be crucial for patients with T2D [8-10]. The American Academy of Sleep Medicine (AASM) clinical practice guidelines suggest that screening questionnaires and facility-based polysomnography (PSG) can be used to screen and diagnose OSA in adult patients with suspected OSA [11]. However, due to the lower specificity and accuracy, screening 
questionnaires can just be used to preliminary screen OSA for suspected sleep disorder. Moreover, the usage of PSG was comparatively expensive and time-wasting. The PSG was also impractical for the clinical evaluation of large atrisk populations [12]. Given the above factors, comprehensive analysis of the molecular mechanism under OSA conditions is so paramount, which contributes to the detection and diagnosis of this sleep disorder in patients with T2D.

Cathepsin S (CTSS), a cysteine protease, participated in antigen presentation and cellular proteolysis $[13,14]$. Moreover, prior studies have proposed a causal relationship between CTSS activity and inflammatory reaction; higher circulating levels of CTSS are associated with increased inflammatory activity $[15,16]$. Experimental studies suggest that CTSS activity is involved in prediabetic alterations in the liver and autoimmune diabetes $[17,18]$. Moreover, CTSS activity has been implicated to play an active role in inflammatory reactions and is upregulated in hypoxic conditions $[19,20]$.

Based on previous experimental reports suggesting a role for CTSS activity in the inflammatory activity in T2D and hypoxic conditions, we hypothesized that plasma levels of CTSS would be a biomarker for diagnosing OSA in patients with T2D. Accordingly, we investigated the association between plasma levels of CTSS and the occurrence and severity of OSA in a hospital-based population. As a second step, we explored the association between CTSS and OSA in patients with T2D.

\section{Materials and Methods}

2.1. Study Design and Population. The observational crosssectional study was performed in Beijing Anzhen Hospital. The study protocol was supported by the Medicine Ethics Committee of Beijing Anzhen Hospital and complied with the Declaration of Helsinki. This study was registered with the Chinese Clinical Trial Registry (No. ChiCTRROC17011027). In total, 180 participants were recruited and underwent overnight full PSG in the Sleep Laboratory between March 2017 and November 2018. Patients with respiratory disease, type 1 diabetes, gestational diabetes, coronary heart disease, hypertension, hyperlipidemia, active hepatitis, cancer, and drug or alcohol addiction were excluded from this study, or other known major diseases were precluded from the study [21]. Participants who were younger than 18 years, refused to sign the consent form, or have incomplete information were also excluded. Finally, we selected a total of 158 eligible participants. Data on participants' baseline demographics were collected from the questionnaires. Anthropometrical characteristics included weight (kilograms), height (meters), body mass index (BMI) $\left(\mathrm{kg} / \mathrm{m}^{2}\right)$, blood pressure, and left ventricular ejection fraction which were calculated by standardized instructions. Current smokers were defined as participants who were smoking or stopped smoking for less than one year before enrollment in this study. Drinkers were participants who consumed alcohol more than three days a week. Serum lipid, high-sensitivity C-reactive protein (hs-CRP), and other indicators were measured as previously described [22, 23].
2.2. OSA Assessment. Each eligible subject underwent a comprehensive sleep assessment. Neck circumference $(\mathrm{cm})$ was measured between the midcervical vertebra and midanterior neck by a flexible tape [24]. The Epworth Sleepiness Scale (ESS) was used to assess the risk of daytime sleepiness, which was measured for each study subject [25]. All participants underwent overnight PSG (SOMNOscreen; SOMNOmedics $\mathrm{GmbH}$, Germany) supported by the US Food and Drug Administration. Alcohol or sleeping medicines must be strictly prohibited before the PSG examination. The SOMNOscreen device continuously monitored and recorded abdominal and chest motion, respiratory effort airflow, arterial oxygen saturation, electroencephalogram, and electrocardiogram through computerized polysomnograms. Based on the 2012 standards of the AASM criteria, sleep stage and cardiopulmonary events were manually evaluated by certified technicians [26]. The definition of hypopnea was greater than $3 \%$ oxygen desaturation and sustaining for at least $10 \mathrm{~s}$. The definition of apnea was airflow complete cessation or a $90 \%$ airflow decrease in baseline amplitude sustaining for at least $10 \mathrm{~s}$. The apnea-hypopnea index (AHI) was calculated as the total of hypopneas and apneas per hour of sleep. The criterion of OSA diagnosis was AHI at least 5 events per hour.

2.3. T2D Assessment. The diagnosis of T2D was based on the criteria established by the WHO in 2011 [27]. Participants with fasting blood glucose $(\mathrm{FBG}) \geq 7 \mathrm{mmol} / \mathrm{L}$, glycated hemoglobin $(\mathrm{HbA} 1 \mathrm{c}) \geq 6.5 \%, 2 \mathrm{~h}$ postload plasma glucose $\geq 11.1 \mathrm{mmol} / \mathrm{L}$, or treatment with antidiabetes medication were regarded as $\mathrm{T} 2 \mathrm{D}$.

2.4. Collection and Storage of Plasma Sample. We quickly obtained fasting blood samples after the PSG examination. Procedures for plasma collection, processing, and storage have been previously described $[22,23]$.

2.5. Luminex Assays. The Magnetic Luminex ${ }^{\circledR}$ Assays were magnetic bead-based antibody microarrays that can be used to facilitate the simultaneous quantitation of up to 100 soluble analytes in a single sample [28]. In the study, we custommade a Luminex panel including CTSS to assess the impact of OSA in T2D (R\&D Systems, Inc., Minneapolis, MN, USA). To ensure the accuracy and validity of the results, as previously described, we assessed the Luminex results by the intra-assay variability and standard curve [22]. In this study, the intra-assay coefficient of variation (CV\%) of the standard curve was $<4.0 \%$ (see Table S1 in the Supplementary Materials).

2.6. Statistical Analysis. Continuous variables were shown as mean \pm standard deviation or median (interquartile range (IQR)) depending on the results of the Kolmogorov-Smirnov test; categorical variables were presented as the number of cases. Normally distributed continuous variables were analyzed by one-way analysis of variance and adjusted by the Bonferroni correction for multiple comparisons. The Kruskal-Wallis test and Bonferroni post hoc analysis were used to evaluate nonnormally distributed continuous variables. The categorical variables were analyzed by the chi- 
square test with Yates correction. The correlations of continuous variables were analyzed by the Pearson or Spearman correlation coefficient. Multiple linear regression analyses (forced entry method) assessed the influence of variables (age, sex, and other variables with $P<0.10$ in the univariate model analysis) for plasma CTSS concentration. The receiver operating characteristic (ROC) curves, the area under the curve (AUC), and the sensitivity and specificity of optimal cutoff CTSS plasma concentration were calculated and recorded for the prediction of OSA based on CTSS concentrations. It is statistically significant when the $P$ value was less than 0.05 . All data were evaluated by SPSS version 25 software (SPSS Inc., Chicago, IL, USA).

\section{Results and Discussion}

3.1. Baseline Clinical Characteristics of the Study Population. The demographic and sleep data of the 158 individuals involved in this study are shown in Table 1. Participants were divided into four groups of control, non-OSA\&T2D, OSA\&non-T2D, and OSA\&T2D based on the FBG and AHI values. Participants in the four groups were matched by age, sex, and BMI. Compared with the control group, higher levels of the diastolic blood pressure, ESS score, AHI, percentage of cumulative time with oxygen saturation below 90\% (CT90), and arousal index were observed in the OSA\&non-T2D group. The "lowest arterial oxygen saturation $\left(\mathrm{SaO}_{2}\right)$ " and "mean $\mathrm{SaO}_{2}$ " were lower in the OSA\&non-T2D group than in the control group. Participants with OSA\&T2D had significantly higher diastolic blood pressure, neck circumference, ESS score, AHI, CT90, and arousal index compared to the non-OSA\&T2D group. The patients with OSA\&T2D had lower "lowest $\mathrm{SaO}_{2}$ " and "mean $\mathrm{SaO}_{2}$ " than the nonOSA\&T2D patients. There was no significant difference in demographic data between the non-OSA\&T2D group and the control group.

Compared to the control group, the levels of total cholesterol, triglyceride, low-density lipoprotein cholesterol, and uric acid were significantly increased in the OSA\&non-T2D group (Table 2).

3.2. CTSS Levels in the Control Group, the Non-OSA\&T2D Group, and the OSA\&T2D Group. The CTSS levels for the control group, the non-OSA\&T2D group, the OSA\&nonT2D group, and the OSA\&T2D group are shown in Table 2. The CTSS levels were as follows: $1.96 \mathrm{ng} / \mathrm{mL}$ (IQR: 1.35-3.78) in the control group, $6.10 \mathrm{ng} / \mathrm{mL}$ (IQR: $3.37-$ 8.01 ) in the non-OSA\&T2D group, $6.93 \mathrm{ng} / \mathrm{mL}$ (IQR: $5.73-$ 8.18 ) in the OSA\&non-T2D group, and $8.37 \mathrm{ng} / \mathrm{mL}$ (IQR: 6.64-10.08) in the OSA\&T2D group. The intergroup comparison of CTSS levels was analyzed by the Kruskal-Wallis test, which showed a significant difference. Compared with the control group, both the non-OSA\&T2D group and the OSA\&non-T2D group showed higher CTSS levels $(P=0.004$ and $P<0.001$, respectively). Plasma CTSS concentration was significantly higher in subjects with OSA\&T2D than those with non-OSA\&T2D $(P=0.048)$. The OSA\&T2D group had higher CTSS levels than the
OSA\&non-T2D group, but there were no statistically significant differences $(P=0.160)$ (Figure 1).

3.3. Relationship between CTSS and Clinical Data. We used Spearman's correlation to investigate the correlation between the levels of CTSS and clinical data. Plasma CTSS expression in all individuals was closely related to the AHI $(r=0.559$, $P<0.001)$, lowest $\mathrm{SaO}_{2}(r=-0.479, P<0.001)$, mean $\mathrm{SaO}_{2}$ $(r=-0.410, P<0.001)$, CT90 $(r=0.363, P<0.001)$, and arousal index $(r=0.433, P<0.001)$. Moreover, plasma CTSS expression was significantly related to plasma FBG $(r=0.427$ , $P<0.001)$ and HbA1c $(r=0.256, P=0.111)$ (Table 3$)$.

We used multiple linear regression analyses to evaluate the correlation between CTSS and clinical and biological parameters. As shown in Table 4, with demographics (age, sex, and BMI) and biological parameters (uric acid, alanine aminotransferase (ALT), and $\gamma$-glutamyl transferase (GGT)) as covariates, it was found that plasma CTSS concentration was positively correlated with the AHI (Beta: 0.386, 95\% confidence interval (CI): 21.988 to $57.781 ; P<0.001$ ), which represents the severity of OSA in this study. In addition, plasma CTSS expression was also positively correlated with the FBG (Beta: 0.340, 95\% CI: 328.871 to $921.777 ; P<$ 0.001).

3.4. ROC Curve Analysis of CTSS Levels for the Prediction Value of OSA. We determined based on ROC curves analysis the optimal threshold value for the optimal meeting point between having the greatest sensitivity and specificity for predicting the occurrence of OSA (Figure 2). The optimal cutoff value of plasma CTSS for the diagnosis of OSA was $5.07 \mathrm{ng} / \mathrm{mL}$ with a corresponding sensitivity of $90.00 \%$ and specificity of $74.10 \%$. The area under the curve was equal to $0.868(P<0.001)$, suggesting that CTSS could be a potential indicator of OSA.

3.5. Discussion. In this research, we demonstrated that patients with OSA\&T2D have significantly elevated plasma CTSS levels, $\approx 1.5$-fold greater than non-OSA\&T2D patients. Plasma CTSS expression was positively correlated to the AHI after adjustment of confounding factors. In addition, after adjustment for possible confounders, plasma CTSS levels remained independently interrelated to the FBG. Plasma CTSS was highly discriminatory accurate in predicting OSA. The evidence demonstrated that increased plasma CTSS could be correlated to an inflammatory reaction, OSA severity, and plasma CTSS as a possible prognostic biomarker with a positive diagnostic value on diagnosing OSA.

In addition, our study showed that serum uric acid expression was significantly higher in the OSA\&non-T2D group than in the control group, which was in accord with the previous research $[29,30]$. Meanwhile, serum uric acid levels were significantly reduced after OSA treatment with continuous positive airway pressure or weight reduction [31]. This finding suggested that it is necessary to diagnose and treat OSA in the early stage.

The relationship between OSA and T2D is a complex interaction. It is established that OSA severity is being 
TABLE 1: Baseline clinical characteristics of the study population.

\begin{tabular}{|c|c|c|c|c|c|}
\hline Parameters & Control $(n=43)$ & Non-OSA\&T2D $(n=15)$ & OSA\&non-T2D $(n=66)$ & OSA\&T2D $(n=34)$ & $P$ value \\
\hline Age (years) & $50.0(38.0-64.0)$ & $58.0(54.0-60.0)$ & $51.0(46.0-56.5)$ & $54.0(46.5-57.0)$ & 0.055 \\
\hline Male, $n(\%)$ & $28(65.1)$ & $10(66.7)$ & $51(77.3)$ & $28(82.4)$ & 0.287 \\
\hline BMI $\left(\mathrm{kg} / \mathrm{m}^{2}\right)$ & $24.6(22.2-29.0)$ & $24.3(21.9-26.4)$ & $26.6(24.2-28.8)$ & $26.7(24.0-28.4)$ & 0.095 \\
\hline SBP (mmHg) & $120.0(114.0-134.0)$ & $115.0(109.0-130.0)$ & $129.0(117.8-137.3)$ & $128.5(119.0-139.3)$ & 0.067 \\
\hline $\mathrm{DBP}(\mathrm{mmHg})$ & $75.0(69.0-85.0)$ & $70.0(65.0-78.0)$ & $83.0(75.0-92.3)^{\S}$ & $82.0(77.8-89.0)^{\dagger \dagger}$ & $<0.001$ \\
\hline Current smoker, $n(\%)$ & $11(25.6)$ & $5(33.3)$ & $20(30.3)$ & $17(50.0)$ & 0.129 \\
\hline Drinker, $n(\%)$ & $5(11.6)$ & $4(26.7)$ & $16(24.2)$ & $11(32.4)$ & 0.147 \\
\hline $\operatorname{LVEF}(\%)$ & $60.0(60.0-68.0)$ & $65.0(62.0-68.0)$ & $67.0(63.0-70.0)^{\S \S}$ & $64.0(59.5-68.0)^{\mathfrak{E}}$ & 0.002 \\
\hline Neck circumference $(\mathrm{cm})$ & $39.0(36.0-41.0)$ & $35.0(33.8-42.0)$ & $41.0(37.0-43.0)$ & $43.5(41.3-46.0)^{\dagger \dagger \dagger \hat{E}}$ & $<0.001$ \\
\hline ESS score & $0.0(0.0-3.0)$ & $6.0(0.0-9.0)$ & $11.0(8.0-15.0)^{\S \S \S}$ & $11.0(7.8-15.0)^{\dagger \dagger}$ & $<0.001$ \\
\hline AHI (events/h) & $3.1(2.1-3.9)$ & $3.5(2.3-7.3)$ & $38.5(26.2-59.6)^{\S \S \S}$ & $47.5(29.6-73.1)^{\dagger \dagger \dagger}$ & $<0.001$ \\
\hline Lowest $\mathrm{SaO}_{2}(\%)$ & $92.0(91.0-93.0)$ & $92.0(88.0-93.0)$ & $80.0(70.0-86.3)^{\S \S \S}$ & $82.0(68.5-86.3)^{\dagger \dagger \dagger}$ & $<0.001$ \\
\hline Mean $\mathrm{SaO}_{2}(\%)$ & $97.0(95.8-98.0)$ & $96.0(95.0-96.0)$ & $94.0(90.0-95.1)^{\S \S \S}$ & $93.5(92.0-95.0)^{\dagger \dagger}$ & $<0.001$ \\
\hline СТ90 (\%) & $0.0(0.0-0.0)$ & $0.0(0.0-0.2)$ & $6.4(0.5-16.8)^{\S \S \S}$ & $4.9(0.2-20.6)^{\dagger \dagger}$ & $<0.001$ \\
\hline Arousal index (events/h) & $0.7(0.0-3.2)$ & $3.5(0.3-6.7)$ & $36.7(12.3-58.1)^{\S \S \S}$ & $11.9(7.2-16.7)^{\dagger}$ & $<0.001$ \\
\hline
\end{tabular}

Data are presented as median (interquartile range) or $n$ (\%), unless otherwise stated. ${ }^{\$ \varsigma \$} P<0.001,{ }^{\$ \S} P<0.01$, and ${ }^{\S} P<0.05$ between OSA\&non-T2D and control. ${ }^{\dagger \dagger} P<0.001,{ }^{\dagger \dagger} P<0.01$, and ${ }^{\dagger \dagger} P<0.05$ between OSA\&T2D and non-OSA\&T2D. ${ }^{\mathfrak{E E E}} P<0.001$, ${ }^{\mathfrak{E}} P<0.01$, and ${ }^{\mathfrak{E}} P<0.05$ between OSA\&T2D and OSA\&non-T2D. OSA: obstructive sleep apnea; T2D: type 2 diabetes; BMI: body mass index; SBP: systolic blood pressure; DBP: diastolic blood pressure; LVEF: left ventricular ejection fraction; ESS: Epworth Sleepiness Scale; AHI: apnea-hypopnea index; $\mathrm{SaO}_{2}$ : arterial oxygen saturation; CT90: percentage of cumulative time with oxygen saturation below $90 \%$.

TABLE 2: Biological parameters and CTSS plasma levels of the study population.

\begin{tabular}{|c|c|c|c|c|c|}
\hline Parameters & Control $(n=43)$ & Non-OSA\&T2D $(n=15)$ & OSA\&non-T2D $(n=66)$ & OSA\&T2D $(n=34)$ & $P$ value \\
\hline $\mathrm{FBG}(\mathrm{mmol} / \mathrm{L})$ & $5.1(4.9-5.3)$ & $6.1(4.9-6.7)^{*}$ & $5.2(5.0-5.6)$ & $7.4(6.0-9.5)^{\mathfrak{E A E}}$ & $<0.001$ \\
\hline HbAlc (\%) & $5.6(5.4-6.0)$ & $6.6(6.1-7.3)$ & $5.7(5.3-5.9)$ & $7.1(6.5-8.5)^{£}$ & $<0.001$ \\
\hline GPS (\%) & $13.7(12.6-14.5)$ & $17.4(15.3-21.4)^{* *}$ & $12.8(11.9-13.6)$ & $17.6(15.0-21.3)^{\mathfrak{E E E}}$ & $<0.001$ \\
\hline Duration of T2D (years) & l & $24.0(14.0-72.0)$ & 1 & $36.0(18.0-88.0)$ & 0.462 \\
\hline Total cholesterol (mmol/L) & $4.6(4.1-5.2)$ & $4.1(3.5-4.9)$ & $5.2(4.6-6.0)^{\S \S}$ & $4.6(3.8-5.7)^{\mathfrak{E}}$ & $<0.001$ \\
\hline Triglyceride $(\mathrm{mmol} / \mathrm{L})$ & $1.1(0.8-1.8)$ & $1.3(0.9-1.4)$ & $1.6(1.2-2.2)^{\S}$ & $1.7(1.3-2.1)$ & 0.001 \\
\hline $\mathrm{HDL}-\mathrm{C}(\mathrm{mmol} / \mathrm{L})$ & $1.2(1.1-1.4)$ & $1.2(1.0-1.6)$ & $1.2(1.0-1.3)$ & $1.1(0.9-1.3)$ & 0.231 \\
\hline LDL-C (mmol/L) & $2.7(2.3-3.2)$ & $2.4(1.7-3.3)$ & $3.3(2.9-3.9)^{\S \S}$ & $2.7(2.1-3.5)^{\mathfrak{E}}$ & $<0.001$ \\
\hline hs-CRP (mg/L) & $0.8(0.3-2.1)$ & $2.0(0.6-7.0)$ & $1.3(0.5-2.3)$ & $1.7(1.0-4.7)$ & 0.018 \\
\hline Platelet (G/L) & $216.5(176.3-262.8)$ & $228.0(171.0-280.0)$ & $226.0(198.0-257.0)$ & $212.0(188.5-260.5)$ & 0.777 \\
\hline Leukocyte (G/L) & $5.6(4.8-7.1)$ & $6.3(5.2-7.9)$ & $6.1(5.3-7.3)$ & $6.9(5.5-7.7)$ & 0.289 \\
\hline Uric acid $(\mu \mathrm{mol} / \mathrm{L})$ & $343.4(294.5-397.3)$ & $337.6(257.4-386.4)$ & $399.0(330.8-484.3)^{\S}$ & $393.9(342.2-472.7)$ & 0.001 \\
\hline $\operatorname{ALT}(\mathrm{U} / \mathrm{L})$ & $20.5(12.8-31.3)$ & $25.0(20.0-41.0)$ & $26.0(16.0-36.5)$ & $29.0(16.5-43.5)$ & 0.082 \\
\hline GGT (U/L) & $23.0(16.0-35.0)$ & $28.5(21.5-48.5)$ & $31.5(20.0-48.5)$ & $41.5(27.0-63.0)$ & 0.004 \\
\hline Homocysteine $(\mu \mathrm{mol} / \mathrm{L})$ & $10.2(8.5-15.4)$ & $12.7(8.9-15.7)$ & $12.0(9.3-15.7)$ & $10.7(8.8-16.3)$ & 0.857 \\
\hline Urea nitrogen $(\mathrm{mmol} / \mathrm{L})$ & $4.7(4.1-5.9)$ & $5.9(4.2-7.8)$ & $5.0(4.5-5.9)$ & $5.0(4.2-6.3)$ & 0.354 \\
\hline Creatinine $(\mu \mathrm{mol} / \mathrm{L})$ & $65.9(54.1-74.8)$ & $59.7(58.4-64.2)$ & $70.7(60.8-78.9)$ & $64.1(57.3-76.4)$ & 0.027 \\
\hline $\operatorname{ALP}(\mathrm{U} / \mathrm{L})$ & $73.0(63.0-90.0)$ & $72.0(60.0-91.8)$ & $72.0(61.5-84.5)$ & $78.0(62.0-88.5)$ & 0.919 \\
\hline $\operatorname{AST}(\mathrm{U} / \mathrm{L})$ & $20.0(18.0-24.3)$ & $21.0(16.0-35.0)$ & $22.0(19.0-26.0)$ & $22.0(18.5-31.5)$ & 0.522 \\
\hline Creatine kinase (U/L) & $96.0(70.0-121.0)$ & $66.0(54.0-85.5)$ & $110.0(70.0-145.0)$ & $94.0(71.0-128.5)$ & 0.071 \\
\hline CTSS (ng/mL) & $1.96(1.35-3.78)$ & $6.10(3.37-8.01)^{* *}$ & $6.93(5.73-8.18)^{\S \varsigma \S}$ & $8.37(6.64-10.08)^{\dagger}$ & $<0.001$ \\
\hline
\end{tabular}

Data are presented as median (interquartile range). ${ }^{* * *} P<0.001,{ }^{* *} P<0.01$, and ${ }^{*} P<0.05$ between non-OSA\&T2D and control. ${ }^{\$ \$ \$} P<0.001,{ }^{\$ \varsigma} P<0.01$, and ${ }^{\S} P<0.05$ between OSA\&non-T2D and control. ${ }^{\dagger \dagger} P<0.001,{ }^{\dagger \dagger} P<0.01$, and ${ }^{\dagger} P<0.05$ between OSA\&T2D and non-OSA\&T2D. ${ }^{\mathfrak{E E E}} P<0.001$, ${ }^{\mathrm{EE}} P<0.01$, and ${ }^{E} P<0.05$ between OSA\&T2D and OSA\&non-T2D. OSA: obstructive sleep apnea; T2D: type 2 diabetes; FBG: fasting blood glucose; HbA1c: glycated hemoglobin; GPS: glycosylated serum protein; HDL-C: high-density lipoprotein cholesterol; LDL-C: low-density lipoprotein cholesterol; hs-CRP: highsensitivity C-reactive protein; ALT: alanine aminotransferase; GGT: $\gamma$-glutamyl transferase; ALP: alkaline phosphatase; AST: aspartate aminotransferase; CTSS: cathepsin S. 


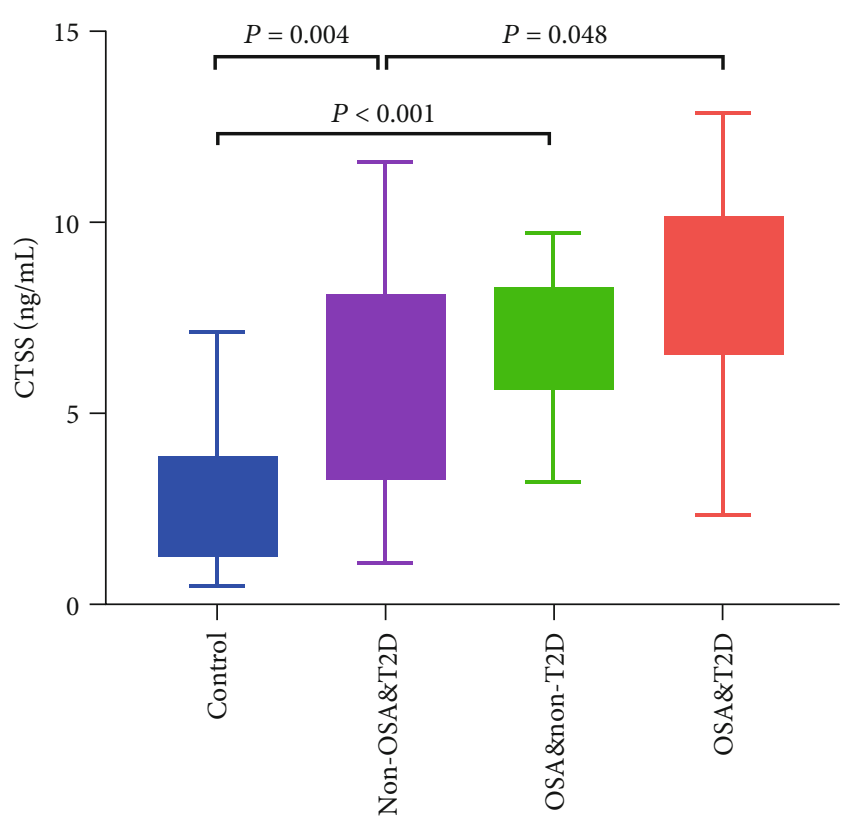

Figure 1: Plasma CTSS levels in the control group, the nonOSA\&T2D group, the OSA\&non-T2D group, and the OSA\&T2D group. Data are median (interquartile range). CTSS: cathepsin S; OSA: obstructive sleep apnea; T2D: type 2 diabetes.

TABLE 3: Correlation between CTSS and clinical and laboratory variables.

\begin{tabular}{lcc}
\hline Parameters & Rho & $P$ value \\
\hline AHI & 0.559 & $<0.001$ \\
Lowest $\mathrm{SaO}_{2}$ & -0.479 & $<0.001$ \\
Mean $\mathrm{SaO}_{2}$ & -0.410 & $<0.001$ \\
CT90 & 0.363 & $<0.001$ \\
Arousal index & 0.433 & $<0.001$ \\
FBG & 0.427 & $<0.001$ \\
HbA1c & 0.256 & 0.111 \\
\hline
\end{tabular}

Spearman's rho was used for correlations between CTSS and clinical and laboratory parameters. CTSS: cathepsin S; AHI: apnea-hypopnea index; $\mathrm{SaO}_{2}$ : arterial oxygen saturation; CT90: percentage of cumulative time with oxygen saturation below 90\%; FBG: fasting blood glucose; HbAlc: glycated hemoglobin.

increasingly recognized as a possible factor of glucose intolerance, insulin resistance, and diabetes, possibly via the effect of recurrent hypoxemia, inflammation, sympathetic nervous system activation, reactive oxygen species formation, or insulin resistance $[4,5,32]$. Furthermore, it has been hypothesized that diabetes may enhance the predisposition for OSA, due to diabetes-associated autonomic dysfunction influence ventilatory control by abnormalities in autonomic nervous system activity and enhanced central chemoreceptor-mediated gain $[6,7]$. Individually, both OSA and T2D are the most common disorders and interrelated to the adverse clinical complications that impose a tremendous burden on public health. In addition, multiple epidemiological studies have indicated a high prevalence of undiagnosed OSA in patients with T2D; screening for sleep patterns and OSA could be of great importance for patients with T2D [10,33].
The AASM clinical practice guidelines suggest that OSA is diagnosed based on clinical measures, including the subjective assessment of somnolence and the overnight multichannel PSG [26]. Although some screening questionnaires are simple, relatively easy to implement, they are restricted to poor specificity and accuracy for the diagnosis of OSA. Current OSA diagnoses and severity grading are mainly based on PSG, but it is expensive and time-consuming. It is also impractical for the clinical evaluation of large at-risk populations [12]. Therefore, we explore circulating biomarkers, which could provide important information on clinical significance for diagnosing OSA.

CTSS, a cysteine protease, is expressed in endothelial cells and participated in antigen presentation and extracellular matrix remodeling [34]. Previous studies have reported that higher circulating levels of CTSS are associated with increased inflammatory activity and involved in the early dysregulation of glucose and insulin metabolism $[16,35]$. Experimental studies suggest that CTSS activity is involved in prediabetic alterations in the liver and in autoimmune diabetes; deficiency in CTSS led to a robust reduction in blood glucose and a decrease in diabetes incidence $[17,18]$. Consistent with previous study, we also found an association between elevated plasma levels of CTSS with FBG even after adjustment for age, sex, BMI, AHI, uric acid, ALT, and GGT.

To our knowledge, this study is the first to elucidate the relationship between CTSS and OSA in patients with T2D. However, previous publications have reported associations of CTSS with hypoxia conditions. RNA sequencing analysis showed that CTSS mRNA levels were higher under hypoxic conditions and hypoxia significantly enhanced CTSS activity in aortic endothelial cells [19, 20]. Likewise, in hypoxia-induced rats, CTSS expression progressively upregulated with hypoxia time [36]. These findings were consistent with the related study about human tissue; CTSS upregulated over twofold in the chronic hypoxia muscles of polysaccharide storage myopathy [37]. Although the role of CTSS under OSA-induced $\mathrm{CIH}$ conditions was unknown, CTSS played an active role in inflammatory reactions and was upregulated in hypoxic conditions. In our study, the OSA\&non-T2D group showed higher CTSS levels compared with the control group. Meanwhile, we also found that plasma CTSS was independently correlated to AHI, even though adjustment was made for age, sex, BMI, FBG, uric acid, ALT, and GGT. An expanded evaluation of CTSS extended to the associations between OSA and T2D, suggesting that inflammatory reactions may be a mechanism by which CTSS contributes to increased OSA risk in patients with T2D. In contrast, neither BMI nor uric acid, ALT, and GGT was found to be associated with CTSS. And intriguingly, the discriminatory accuracy of plasma CTSS was high on diagnosing OSA. Given the high prevalence of undiagnosed OSA in patients with T2D, it was available using plasma CTSS to preliminarily diagnosis OSA in patients with T2D, instead of using complicated overnight multichannel PSG. 
TABLE 4: Association between levels of CTSS and variables in univariate and multiple linear regression models.

\begin{tabular}{|c|c|c|c|c|c|c|}
\hline & \multicolumn{3}{|c|}{ Univariate models } & \multicolumn{3}{|c|}{ Multiple model $\left(R^{2}=0.373, P<0.001\right)$} \\
\hline & Beta & $95 \% \mathrm{CI}$ & $P$ value & Beta & $95 \%$ CI & $P$ value \\
\hline Age (years) & -0.005 & -51.183 to 48.343 & 0.955 & 0.034 & -33.850 to 54.399 & 0.645 \\
\hline Sex (female vs. male) & 0.186 & 124.692 to 2497.537 & 0.031 & 0.066 & -603.815 to 1524.928 & 0.393 \\
\hline $\operatorname{BMI}\left(\mathrm{kg} / \mathrm{m}^{2}\right)$ & 0.116 & -34.536 to 227.965 & 0.148 & -0.054 & -189.882 to 89.546 & 0.478 \\
\hline $\operatorname{LVEF}(\%)$ & 0.022 & -69.687 to 91.954 & 0.786 & & & \\
\hline AHI (events/h) & 0.506 & 39.757 to 69.073 & $<0.001$ & 0.386 & 21.988 to 57.781 & $<0.001$ \\
\hline $\mathrm{FBG}(\mathrm{mmol} / \mathrm{L})$ & 0.444 & 602.300 to 1165.896 & $<0.001$ & 0.340 & 328.871 to 921.777 & $<0.001$ \\
\hline Total cholesterol $(\mathrm{mmol} / \mathrm{L})$ & 0.075 & -194.509 to 541.078 & 0.353 & & & \\
\hline Triglyceride $(\mathrm{mmol} / \mathrm{L})$ & 0.085 & -158.839 to 527.189 & 0.291 & & & \\
\hline LDL-C (mmol/L) & 0.095 & -204.688 to 815.319 & 0.239 & & & \\
\hline hs-CRP (mg/L) & 0.074 & -59.811 to 161.904 & 0.364 & & & \\
\hline Uric acid $(\mu \mathrm{mol} / \mathrm{L})$ & 0.136 & -0.594 to 8.440 & 0.088 & -0.095 & -7.434 to 1.876 & 0.239 \\
\hline ALT (U/L) & 0.171 & 1.374 to 40.573 & 0.036 & 0.012 & -17.892 to 20.757 & 0.883 \\
\hline GGT (U/L) & 0.208 & 4.290 to 29.517 & 0.009 & 0.113 & -3.932 to 21.711 & 0.172 \\
\hline Creatinine $(\mu \mathrm{mol} / \mathrm{L})$ & 0.087 & -9.832 to 33.883 & 0.279 & & & \\
\hline Creatine kinase (U/L) & 0.013 & -3.387 to 3.966 & 0.877 & & & \\
\hline
\end{tabular}

The multiple linear regression model includes age, sex, BMI, and other variables with $P<0.10$ in univariate model analysis. $R^{2}$ : adjusted $R^{2}$ of the multiple linear regression model; CI: confidence intervals; CTSS: cathepsin S; BMI: body mass index; LVEF: left ventricular ejection fraction; AHI: apnea-hypopnea index; FBG: fasting blood glucose; LDL-C: low-density lipoprotein cholesterol; hs-CRP: high-sensitivity C-reactive protein; ALT: alanine aminotransferase; GGT: $\gamma$ glutamyl transferase.

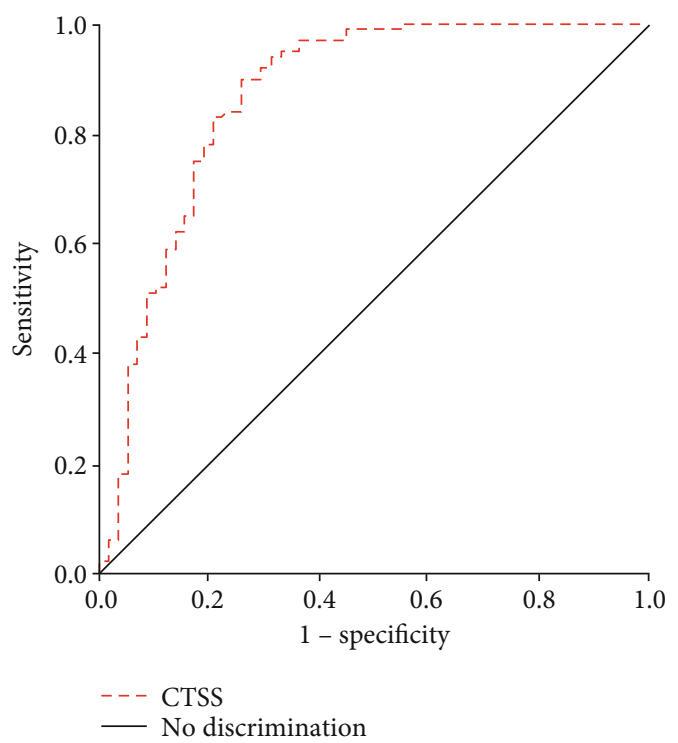

FIGURE 2: Receiver operating characteristic curves using CTSS levels for prediction of OSA (AUC: 0.868, 95\% CI: 0.802-0.934, $P<0.001$; sensitivity: $90.00 \%$, specificity: $74.10 \%)$. CTSS: cathepsin S; OSA: obstructive sleep apnea; AUC: area under the curve; CI: confidence intervals.

To avoid bias in this research, the Luminex experiment was conducted in light of the manufacturer's instructions by the experimenter who does not know the patients' clinical information. Moreover, the confounding effects of risk factors for plasma CTSS levels and AHI were adjusted by multiple linear regression analyses. In addition, plasma CTSS may provide vital value to screen and diagnose OSA in patients with T2D, having important clinical significance. However, the limitations should be noted in our research. First, this study was an observational cross-sectional research, which only showed a correlated relationship. Second, the number of the study population was relatively small; hence, multicenter studies with larger sample sizes were warranted.

\section{Conclusions}

In conclusion, we demonstrated that plasma CTSS expression was significantly elevated in patients with OSA\&T2D and was associated with the AHI, which represents the severity of OSA in this study. Plasma CTSS levels provided high discriminatory accuracy values on diagnosing OSA; it might be a potential biomarker with a positive diagnostic value for inflammatory reactions in patients with OSA\&T2D. Further, large-scale and prospective studies are explored to verify these findings.

\section{Data Availability}

The datasets used and analyzed during the current study are available from the corresponding author on reasonable request.

\section{Conflicts of Interest}

All authors do not have any conflicts of interest.

\section{Acknowledgments}

The study was supported by the International Science and Technology Cooperation Programme of China 
(2015DFA30160), National Natural Science Foundation of China (81470567 and 81670322), Beijing Municipal Science \& Technology Commission (Z141100006014057 and Z181100006218133), and Beijing Municipal Natural Science Foundation (7182047).

\section{Supplementary Materials}

Table S1: evaluation of Luminex assay standard curves and intra-assay variability for CTSS levels determined using the Luminex. (Supplementary Materials)

\section{References}

[1] S. Javaheri, F. Barbe, F. Campos-Rodriguez et al., "Sleep apnea," Journal of the American College of Cardiology, vol. 69, no. 7, pp. 841-858, 2017.

[2] N. A. Dewan, F. J. Nieto, and V. K. Somers, "Intermittent hypoxemia and OSA: implications for comorbidities," Chest, vol. 147, no. 1, pp. 266-274, 2015.

[3] J. A. Dempsey, S. C. Veasey, B. J. Morgan, and C. P. O'Donnell, "Pathophysiology of sleep apnea," Physiological Reviews, vol. 90, no. 1, pp. 47-112, 2010.

[4] N. Iiyori, L. C. Alonso, J. Li et al., "Intermittent hypoxia causes insulin resistance in lean mice independent of autonomic activity," American Journal of Respiratory and Critical Care Medicine, vol. 175, no. 8, pp. 851-857, 2007.

[5] A. Subramanian, N. J. Adderley, A. Tracy et al., "Risk of incident obstructive sleep apnea among patients with type 2 diabetes," Diabetes Care, vol. 42, no. 5, pp. 954-963, 2019.

[6] T. Huang, B. M. Lin, M. J. Stampfer, S. S. Tworoger, F. B. Hu, and S. Redline, "A population-based study of the bidirectional association between obstructive sleep apnea and type 2 diabetes in three prospective U.S. cohorts," Diabetes Care, vol. 41, no. 10, pp. 2111-2119, 2018.

[7] R. N. Aurora and N. M. Punjabi, "Obstructive sleep apnoea and type 2 diabetes mellitus: a bidirectional association," The Lancet. Respiratory medicine, vol. 1, no. 4, pp. 329-338, 2013.

[8] R. S. Aronsohn, H. Whitmore, E. van Cauter, and E. Tasali, "Impact of untreated obstructive sleep apnea on glucose control in type 2 diabetes," American Journal of Respiratory and Critical Care Medicine, vol. 181, no. 5, pp. 507-513, 2010.

[9] J. E. Heffner, Y. Rozenfeld, M. Kai, E. A. Stephens, and L. K. Brown, "Prevalence of diagnosed sleep apnea among patients with type 2 diabetes in primary care," Chest, vol. 141, no. 6 , pp. 1414-1421, 2012.

[10] A. A. Tahrani, A. Ali, N. T. Raymond et al., "Obstructive sleep apnea and diabetic neuropathy: a novel association in patients with type 2 diabetes," American Journal of Respiratory and Critical Care Medicine, vol. 186, no. 5, pp. 434-441, 2012.

[11] B. Mokhlesi and A. S. Cifu, "Diagnostic testing for obstructive sleep apnea in adults," JAMA, vol. 318, no. 20, pp. 2035-2036, 2017.

[12] W. T. McNicholas, C. L. Bassetti, L. Ferini-Strambi et al., "Challenges in obstructive sleep apnoea," The Lancet Respiratory Medicine, vol. 6, no. 3, pp. 170-172, 2018.

[13] J. P. Liuzzo, S. S. Petanceska, D. Moscatelli, and L. A. Devi, "Inflammatory mediators regulate cathepsin $\mathrm{S}$ in macrophages and microglia: a role in attenuating heparan sulfate interactions," Molecular Medicine, vol. 5, no. 5, pp. 320-333, 1999.
[14] T. Y. Nakagawa, W. H. Brissette, P. D. Lira et al., "Impaired invariant chain degradation and antigen presentation and diminished collagen-induced arthritis in cathepsin S null mice," Immunity, vol. 10, no. 2, pp. 207-217, 1999.

[15] S. Taleb, R. Cancello, C. Poitou et al., "Weight loss reduces adipose tissue cathepsin $\mathrm{S}$ and its circulating levels in morbidly obese women," Journal of Clinical Endocrinology \& Metabolism, vol. 91, no. 3, pp. 1042-1047, 2006.

[16] E. Jobs, U. Risérus, E. Ingelsson et al., "Serum cathepsin S is associated with serum C-reactive protein and interleukin-6 independently of obesity in elderly men," Journal of Clinical Endocrinology \& Metabolism, vol. 95, no. 9, pp. 4460-4464, 2010.

[17] L. C. Hsing, E. A. Kirk, T. S. McMillen et al., "Roles for cathepsins $\mathrm{S}, \mathrm{L}$, and B in insulitis and diabetes in the NOD mouse," Journal of Autoimmunity, vol. 34, no. 2, pp. 96-104, 2010.

[18] J. C. Lafarge, M. Pini, V. Pelloux et al., "Cathepsin S inhibition lowers blood glucose levels in mice," Diabetologia, vol. 57, no. 8, pp. 1674-1683, 2014.

[19] K. Stellos, A. Gatsiou, K. Stamatelopoulos et al., "Adenosineto-inosine RNA editing controls cathepsin S expression in atherosclerosis by enabling HuR-mediated post-transcriptional regulation," Nature Medicine, vol. 22, no. 10, pp. 1140-1150, 2016.

[20] A. Moldobaeva and E. M. Wagner, "Difference in proangiogenic potential of systemic and pulmonary endothelium: role of CXCR2," American Journal of Physiology-Lung Cellular and Molecular Physiology, vol. 288, no. 6, pp. L1117-L1123, 2005.

[21] C. A. Kushida, D. A. Nichols, S. F. Quan et al., "The apnea positive pressure long-term efficacy study (APPLES): rationale, design, methods, and procedures," Journal of Clinical Sleep Medicine, vol. 2, no. 3, pp. 288-300, 2006.

[22] W. W. Wen, Y. Ning, Q. Zhang et al., "TNFRSF11B: a potential plasma biomarker for diagnosis of obstructive sleep apnea," Clin Chim Acta, vol. 490, pp. 39-45, 2019.

[23] W. W. Wen, H. L. Sun, Y. X. Yang et al., "The association between circulating APRIL levels and severity of obstructive sleep apnea in Chinese adults," Clin Chim Acta, vol. 508, pp. 161-169, 2020.

[24] R. J. Davies and J. R. Stradling, "The relationship between neck circumference, radiographic pharyngeal anatomy, and the obstructive sleep apnoea syndrome," European Respiratory Journal, vol. 3, no. 5, pp. 509-514, 1990.

[25] M. W. Johns, "A new method for measuring daytime sleepiness: the Epworth sleepiness scale," Sleep, vol. 14, no. 6, pp. 540-545, 1991.

[26] R. B. Berry, R. Budhiraja, D. J. Gottlieb et al., "Rules for scoring respiratory events in sleep: update of the 2007 AASM Manual for the Scoring of Sleep and Associated Events. Deliberations of the Sleep Apnea Definitions Task Force of the American Academy of Sleep Medicine," Journal of Clinical Sleep Medicine, vol. 8, no. 5, pp. 597-619, 2012.

[27] R. Kumar, L. P. Nandhini, S. Kamalanathan, J. Sahoo, and M. Vivekanadan, "Evidence for current diagnostic criteria of diabetes mellitus," World Journal of Diabetes, vol. 7, no. 17, pp. 396-405, 2016.

[28] A. Leligdowicz, A. L. Conroy, M. Hawkes et al., "Validation of two multiplex platforms to quantify circulating markers of inflammation and endothelial injury in severe infection," PLoS One, vol. 12, no. 4, p. e0175130, 2017. 
[29] A. Kanbay, H. Inonu, Y. Solak et al., "Uric acid as a potential mediator of cardiovascular morbidity in obstructive sleep apnea syndrome," European Journal of Internal Medicine, vol. 25, no. 5, pp. 471-476, 2014.

[30] C. Zheng, H. Song, S. Wang et al., "Serum uric acid is independently associated with risk of obstructive sleep apneahypopnea syndrome in Chinese patients with type 2 diabetes," Disease Markers, vol. 2019, Article ID 4578327, 6 pages, 2019.

[31] P. Steiropoulos, I. Kotsianidis, E. Nena et al., "Long-term effect of continuous positive airway pressure therapy on inflammation markers of patients with obstructive sleep apnea syndrome," Sleep, vol. 32, no. 4, pp. 537-543, 2009.

[32] A. Lecube, R. Simó, M. Pallayova et al., "Pulmonary function and sleep breathing: two new targets for type 2 diabetes care," Endocrine Reviews, vol. 38, no. 6, pp. 550-573, 2017.

[33] K. Spiegel, E. Tasali, R. Leproult, and E. van Cauter, "Effects of poor and short sleep on glucose metabolism and obesity risk," Reviews Endocrinology, vol. 5, no. 5, pp. 253-261, 2009.

[34] J. Reiser, B. Adair, and T. Reinheckel, "Specialized roles for cysteine cathepsins in health and disease," Journal of Clinical Investigation, vol. 120, no. 10, pp. 3421-3431, 2010.

[35] E. Jobs, U. Riserus, E. Ingelsson et al., "Serum cathepsin S is associated with decreased insulin sensitivity and the development of type 2 diabetes in a community-based cohort of elderly men," Diabetes Care, vol. 36, no. 1, pp. 163-165, 2012.

[36] S. Otsuki, H. Sawada, N. Yodoya et al., "Potential contribution of phenotypically modulated smooth muscle cells and related inflammation in the development of experimental obstructive pulmonary vasculopathy in rats," PLoS ONE, vol. 10, no. 2, p. e0118655, 2015.

[37] E. Barrey, E. Mucher, N. Jeansoule et al., "Gene expression profiling in equine polysaccharide storage myopathy revealed inflammation, glycogenesis inhibition, hypoxia and mitochondrial dysfunctions," BMC Veterinary Research, vol. 5, no. 1, p. 29, 2009. 